\title{
Reflection and Refraction of Plane Harmonic Waves at an Interface Between Elastic Solid and Magneto-thermoelastic Diffusion Solid with Voids
}

\author{
Rajneesh Kumar ${ }^{1}$, Tarun Kansal ${ }^{2}$ \\ ${ }^{1}$ Department of Mathematics, Kurukshetra University \\ Kurukshetra-136119, India \\ E-mail: rajneesh_kuk@rediffmail.com \\ ${ }^{2}$ Department of Mathematics, M.N.College, Shahabad (M.)-136135, India \\ E-mail: tarun1_kansal@yahoo.co.in-correspondingauthor
}

Received: 21 July 2016; revised: 23 January 2017; accepted: 24 January 2017; published online: 16 March 2017

\begin{abstract}
The problem of the reflection and refraction phenomenon due to longitudinal and transverse waves incident obliquely at a plane interface between uniform elastic solid half-space and magneto-thermoelastic diffusive solid half-space with voids has been studied. It is found that the amplitude ratios of various reflected and refracted waves are functions of the angle of incidence and frequency of the incident wave. The amplitude ratios and energy ratios have been computed numerically for a particular model. The variations of energy ratios with angle of incidence are shown graphically.
\end{abstract}

Key words: magneto-thermoelastic diffusive solid, elastic waves, reflection, refraction, amplitude ratios, energy ratios

\section{INTRODUCTION}

In classical theory of thermoelasticity, Fourier's heat conduction theory assumes that the thermal disturbances propagate at infinite speed, which is unrealistic from the physical point of view. Two different generalizations of the classical theory of thermoelasticity have been developed which predict only finite velocity of propagation for heat and displacement fields. The first one, given by Lord and Shulman [1], incorporates a flux rate term into the Fourier's law of heat conduction and formulates a generalized theory admitting finite speed for thermal signals. The second, given by Green and Lindsay [2], develops a temperature rate dependent thermoelasticity by including temperature rate among the constitutive variables, which does not violate the classical Fourier's law of heat conduction. Lord and Shulman [1] theory of generalized thermoelasticity has been further extended to homogeneous anisotropic heat conducting materials recommended by Dhaliwal and Sherief [3]. All these theories predict a finite speed of heat propagation. Chanderashekhariah [4] refers to this wave like thermal disturbance as second sound. A survey article of various representative theories in the range of generalized thermoelasticity has been brought out by Hetnarski and Ignaczak [5].

The linear theory of elastic materials with voids is an important extension to the classical theory of elasticity. This theory is used for investigating various types of geological and biological materials, where the classical theory of elasticity is not adequate. In this theory, the elastic materials deal with the distribution of small voids or pores in which the void volume is included among the kinematical variables. Nunziato and Cowin [6] developed a nonlinear theory of elastic materials with voids. In fact, the linear theory of elastic material with voids is a special case of the non-linear theory of elastic material with voids. Cowin and Nunziato [7] developed a linear theory of elastic materials with voids. They explained that the linear theory differs significantly from the classical linear elasticity as the volume fraction correspond- 
ing to the void volume is taken as an independent kinematical variable. Iesan [8] developed a theory of thermoelastic material with voids. Ciarletta and Scalia [9] studied the nonlinear theory of nonsimple thermoelastic materials with voids.

Diffusion is defined as the spontaneous movement of the particles from a high concentration region to the low concentration region and it occurs in response to a concentration gradient expressed as the change in the concentration due to change in position. Thermal diffusion utilizes the transfer of heat across a thin liquid or gas to accomplish isotope separation. Today, thermal diffusion remains a practical process to separate isotopes of noble gases (e.g. xexon) and other light isotopes (e.g. carbon) for research purposes. In most of the applications, the concentration is calculated using what is known as Fick's law. This is a simple law which does not take into consideration the mutual interaction between the introduced substance and the medium into which it is introduced or the effect of temperature on this interaction. However, there is a certain degree of coupling with temperature and temperature gradients as temperature speeds up the diffusion process. The thermodiffusion in elastic solids is due to coupling of fields of temperature, mass diffusion and that of strain in addition to heat and mass exchange with the environment.

Nowacki [10-13] developed the theory of thermoelastic diffusion by using a coupled thermoelastic model. Dudziak and Kowalski [14] and Olesiak and Pyryev [15], respectively, discussed the theory of thermodiffusion and coupled quasistationary problems of thermal diffusion for an elastic layer. They studied the influence of cross effects arising from the coupling of the fields of temperature, mass diffusion and strain due to which the thermal excitation results in additional mass concentration and that generates additional fields of temperature. Gawinecki et al.[16] proved a theorem about existence, uniqueness and regularity of the solution to an initialboundary value problem for a nonlinear coupled parabolic system. They used an energy method, method of Sobolev spaces, semigroup theory and Banach fixed point theorem to prove the theorem. Gawinecki and Szymaniec [17] proved a theorem about global existence of the solution to the initialvalue problem for a nonlinear hyperbolic parabolic system of coupled partial differential equation of second order describing the process of thermodiffusion in solid body. Uniqueness and reciprocity theorems for the equations of generalized thermoelastic diffusion problem, in isotropic media, was proved by Sherief et al.[18] on the basis of the variational principle equations, under restrictive assumptions on the elastic coefficients. Due to the inherit complexity of the derivation of the variational principle equations, Aouadi [19] proved this theorem in the Laplace transform domain, under the assumption that the functions of the problem are continuous and the inverse Laplace transform of each is also unique. Sherief and Saleh [20] investigated the problem of a thermoelastic halfspace in the context of the theory of generalized thermoelastic diffusion with one relaxation time. Kumar and Kansal [21] developed the basic equations of anisotropic thermoelastic diffusion based upon Green-Lindsay model and proved the variational principle, uniqueness and reciprocity theorems.

Borejko [22] discussed the reflection and transmission coefficients for three- dimensional plane waves in elastic media. Wu and Lundberg [23] investigated the problem of reflection and transmission of the energy of harmonic elastic waves in a bent bar. Sinha and Elsibai [24] discussed the reflection and refraction of thermoelastic waves at an interface of two semi-infinite media with two relaxation times. Sharma and Gogna [25] discussed the problem of reflection and refraction of plane harmonic waves at an interface between elastic solid and porous solid saturated by viscous liquid. Tomar and Arora [26] studied reflection and transmission of elastic waves at an elastic/porous solid saturated by immiscible fluids. Kumar and Sarthi [27] discussed the reflection and refraction of thermoelastic plane waves at an interface of two thermoelastic media without energy dissipation. Abd-alla and Al-dawy [28] attempted the problem on the reflection phenomena of SV- wave in a generalized thermoelastic medium. They used the generalized thermoelastic waves to study the effects of one or two thermal relaxation times on the reflection plane harmonic waves. Ciarletta and Sumbatyan [29] discussed the problem of the reflection of plane waves by the free boundary of a porous elastic halfspace. Singh and Tomar [30] investigated the problem of the reflection and transmission of transverse waves at a plane interface between two different porous elastic half-spaces. They obtained the reflection and transmission coefficients of the reflected and transmitted waves. Singh [31] investigated the problem of elastic wave propagation in a generalized thermoelastic material with voids using the Lord-Shulman theory. Singh and Tomar [32] attempted the problem of plane waves in thermo-elastic material with voids by using the linear theory of thermo-elastic material with voids developed by Iesan [8]. Das et al.[33] discussed the problem of the reflection of generalized thermoelastic waves from isothermal and insulated boundaries. Singh [34] studied reflection and transmission of couple longitudinal waves at a plane interface between two dissimilar half-spaces of thermo-elastic materials with voids. Kumar and Kumar [35] studied the problem of reflection and transmission at the plane boundary of elastic half-space and initially stressed thermoelastic diffusion with voids half-space.

Abd-alla [36] studied the relaxation effects on the reflection of generalized magneto-thermoelastic waves. Othman and Song [37] discussed the reflection of magnetothermoelastic waves with two relaxation times in an isotropic elastic medium under the effect of reference temperature on the modulus of elasticity. Othman and Kumar [38] investigated the reflection of plane harmonic waves in magneto generalized thermoelasticity theories and obtained the expressions for the reflection coefficients. Abo-Dahab [39] 
studied the propagation of plane waves from the stress free elastic half-space with void, under thermal relaxation and magnetic field. Othman [40] investigated the propagation of electromagneto-thermoelastic disturbances produced by a thermal shock in a finitely conducting elastic half. Singh et al.[41] discussed the problem of reflection of plane waves from a free surface of a generalized magneto-thermoelastic solid half-space with diffusion.

In the present paper, the reflection and refraction phenomenon at a plane interface between an elastic solid medium and a magneto-thermoelastic diffusive solid medium with voids has been analyzed. In magneto-thermoelastic diffusive solid medium with voids, potential functions are introduced to represent four longitudinal waves and one transverse wave. The amplitude ratios of various reflected and refracted waves to that of the incident wave are derived. These amplitude ratios are further used to find the expressions of energy ratios of various reflected and refracted waves to that of the incident wave. The graphical representation is given for these energy ratios for different direction of propagation. The law of conservation of energy at the interface is verified.

\section{BASIC EQUATIONS}

The variations of the magnetic field and electric fields are perfectly slowly moving medium and are given by the Maxwell's equations

$$
\begin{gathered}
\nabla \times \vec{h}=\vec{J}+\epsilon_{0} \frac{\partial \vec{E}}{\partial t}, \\
\nabla \times \vec{E}=-\mu_{0} \frac{\partial \vec{h}}{\partial t}, \\
\vec{E}=-\mu_{0}\left(\frac{\partial \vec{u}}{\partial t} \times \vec{H}\right), \\
\nabla \cdot \vec{h}=0,
\end{gathered}
$$

Maxwell stress components are given by

$$
T_{i j}=\mu_{0}\left(H_{i} h_{j}+H_{j} h_{i}-(\vec{H} \cdot \vec{h}) \delta_{i j}\right),
$$

where $\vec{H}$ is the external applied magnetic field intensity vector, $\vec{h}$ is the induced magnetic field vector, $\vec{E}$ is the induced electric field vector, $\vec{J}$ is the current density vector, $\vec{u}$ is the displacement vector, $\mu_{0}$ is the magnetic permeability, $\epsilon_{0}$ is the electric permittivity, $T_{i j}$ are the components of Maxwell stress tensor, $\delta_{i j}$ is the Kroneker delta and $\nabla=\hat{i} \frac{\partial}{\partial x}+\hat{j} \frac{\partial}{\partial y}+\hat{k} \frac{\partial}{\partial z}$.

The above equations (1)-(5) are supplemented by the field of equations of motion and constitutive relations in the theory of generalized thermoelastic diffusion solid with voids, taking into account the Lorentz force

(i) Constitutive relations

$$
\begin{gathered}
\sigma_{i j}=2 \mu e_{i j}+\delta_{i j}\left[\lambda e_{k k}+\gamma^{*} \phi\right. \\
\left.-\beta_{1}\left(T+\tau_{1} \dot{T}\right)-\beta_{2}\left(C+\tau^{1} \dot{C}\right)\right], \\
g^{*}=-\gamma^{*} e_{k k}-d^{*} \phi+\xi^{*}\left(T+\tau_{1} \dot{T}\right)+\zeta^{*}\left(C+\tau^{1} \dot{C}\right), \\
h_{i}^{*}=a^{*} \phi_{, i} \\
\rho T_{0} S=k+\rho C_{E}(T+\alpha \dot{T})+\xi^{*} T_{0} \phi \\
+\beta_{1} T_{0} e_{k k}+a T_{0}(C+\beta \dot{C}), \\
P=-\beta_{2} e_{k k}-\zeta^{*} \phi+b\left(C+\tau^{1} \dot{C}\right)-a\left(T+\tau_{1} \dot{T}\right),
\end{gathered}
$$

(ii) Equations of motion

$$
\begin{gathered}
\mu u_{i, j j}+(\lambda+\mu) u_{j, i j}+\gamma^{*} \phi_{, i} \\
-\beta_{1}\left(T+\tau_{1} \dot{T}\right)_{, i}-\beta_{2}\left(C+\tau^{1} \dot{C}\right)_{, i}+F_{i}=\rho \ddot{u}_{i}, \\
\quad a^{*} \phi_{, i i}-d^{*} \phi-\gamma^{*} e_{k k} \\
+\xi^{*}\left(T+\tau_{1} \dot{T}\right)+\zeta^{*}\left(C+\tau^{1} \dot{C}\right)=\rho \chi \ddot{\phi},
\end{gathered}
$$

(iii) Equation of heat conduction

$$
\begin{gathered}
\rho C_{E}\left(\dot{T}+\tau_{0} \ddot{T}\right)+\beta_{1} T_{0}\left(\dot{e}_{k k}+\varepsilon \tau_{0} \ddot{e}_{k k}\right) \\
+\xi^{*} T_{0}\left(\dot{\phi}+\varepsilon \tau_{0} \ddot{\phi}\right)+a T_{0}(\dot{C}+\gamma \ddot{C})=K T_{, i i},
\end{gathered}
$$

(iv) Equation of mass diffusion

$$
\begin{gathered}
D \beta_{2} e_{k k, i i}+D \zeta^{*} \phi_{, i i}+D a\left(T+\tau_{1} \dot{T}\right)_{, i i} \\
+\left(\dot{C}+\varepsilon \tau^{0} \ddot{C}\right)-D b\left(C+\tau^{1} \dot{C}\right)_{, i i}=0,
\end{gathered}
$$

where $\beta_{1}=(3 \lambda+2 \mu) \alpha_{t}$ and $\beta_{2}=(3 \lambda+2 \mu) \alpha_{c} ; \lambda, \mu$ are Lame's constants, $\alpha_{t}$ is the coefficient of linear thermal expansion and $\alpha_{c}$ is the coefficient of linear diffusion expansion. $a, b$ are, respectively, coefficients describing the measure of thermodiffusion and of mass diffusion effects, $T=\Theta-T_{0}$ is small temperature increment; $\Theta$ is the absolute temperature of the medium; $T_{0}$ is the reference temperature of the body chosen such that $\left|\frac{T}{T_{0}}\right| \ll 1, C$ is the concentration of the diffusive material in the elastic body. $u_{i}$ are the components of the displacement vector $\vec{u}, \rho$ is the density assumed to be independent of the time, $\sigma_{i j}, e_{i j}\left(=\frac{1}{2}\left(u_{i, j}+u_{j, i}\right)\right)$ are the components of the stress and strain tensors, respectively, $e_{k k}$ is the dilatation, $S$ is the entropy per unit mass, $P$ is the chemical potential per unit mass, $C_{E}$ is the specific heat at the constant strain, $K$ is the coefficient of the thermal conductivity, $D$ is the thermoelastic diffusion constant, $k$ is a material constant. $\phi$ is the volume fraction field, $h_{i}^{*}$ is the equilibrated stress vector, $g^{*}$ is the intrinsic equilibrated body force, $\chi$ is the equilibrated inertia, $a^{*}, d^{*}, \gamma^{*}, \xi^{*}, \zeta^{*}$ are constitutive coefficients. $\tau^{0}, \tau^{1}$ are diffusion relaxation times 
with $\tau^{1} \geq \tau^{0} \geq 0$ and $\tau_{0}, \tau_{1}$ are thermal relaxation times with $\tau_{1} \geq \tau_{0} \geq 0 . F_{i}$ are the components of the Lorentz force $\vec{F}$ given by

$$
\vec{F}=\mu_{0}(\vec{J} \times \vec{H}) .
$$

Here $k=\tau_{1}=\tau^{1}=0, \varepsilon=1, \gamma=\tau_{0}$ for Lord-Shulman (L-S) model and $\varepsilon=0, \gamma=\tau^{0}$ for Green-Lindsay (G-L) model. In the above equations, a comma followed by a suffix denotes spatial derivative and a superposed dot denotes the derivative with respect to time.

The equations of motion in a homogeneous isotropic elastic solid medium are

$$
\mu^{e} u_{i, j j}^{e}+\left(\lambda^{e}+\mu^{e}\right) u_{j, i j}^{e}=\rho^{e} \ddot{u}_{i}^{e},
$$

where $\lambda^{e}, \mu^{e}$ are Lame's constants, $u_{i}^{e}$ are the components of the displacement vector $\vec{u}^{e}, \rho^{e}$ is density corresponding to isotropic elastic solid.

The stress-strain relation in the isotropic elastic solid medium is given by

$$
\sigma_{i j}^{e}=2 \mu^{e} e_{i j}^{e}+\lambda^{e} e_{k k}^{e} \delta_{i j},
$$

where $\sigma_{i j}^{e}, e_{i j}^{e}\left(=\frac{1}{2}\left(u_{i, j}^{e}+u_{j, i}^{e}\right)\right)$ are the components of the stress and strain tensor, respectively, $e_{k k}^{e}$ is the dilatation.

\section{FORMULATION OF THE PROBLEM}

We consider an isotropic elastic solid half-space lying over a homogeneous isotropic, generalized magnetothermoelastic diffusive solid half-space with voids. The origin of the Cartesian coordinate system $\left(x_{1}, x_{2}, x_{3}\right)$ is taken at any point on the plane surface (interface) and $x_{3}$-axis points vertically downwards into the magneto-thermoelastic diffusive solid half-space with voids. The elastic solid half-space occupies the region $x_{3} \leq 0$ (medium I) and the region $x_{3} \geq 0$ is occupied by the dissipative thermoelastic diffusive solid half-space with voids (medium II) permeated with an initial magnetic field $\vec{H}=\left(0, H_{0}, 0\right)$ acting along the $x_{2}-$ axis as shown in Figure 1. We consider plane waves in the $x_{1}-x_{3}$ plane with wave front parallel to the $x_{2}-$ axis. For two dimensional problem, the displacement vectors $\vec{u}^{e}$ in medium I and $\vec{u}$ in medium II are taken as

$$
\vec{u}^{e}=\left(u_{1}^{e}, 0, u_{3}^{e}\right), \vec{u}=\left(u_{1}, 0, u_{3}\right) .
$$

From equations (1)-(3), the components of electric field, magnetic field and current density vectors are

$$
\begin{gathered}
E_{1}=\mu_{0} H_{0} \dot{u}_{3}, E_{2}=0, E_{3}=-\mu_{0} H_{0} \dot{u}_{1}, \\
h_{1}=0, h_{2}=-H_{0} e^{*}, h_{3}=0,
\end{gathered}
$$

$$
\begin{aligned}
& J_{1}=H_{0}\left(\frac{\partial e^{*}}{\partial z}-\varepsilon_{0} \mu_{0} \ddot{u}_{3}\right), \\
& J_{2}=0, \\
& J_{3}=H_{0}\left(-\frac{\partial e^{*}}{\partial x}+\varepsilon_{0} \mu_{0} \ddot{u}_{1}\right),
\end{aligned}
$$

where $e^{*}=\frac{\partial u_{1}}{\partial x_{1}}+\frac{\partial u_{3}}{\partial x_{3}}$.

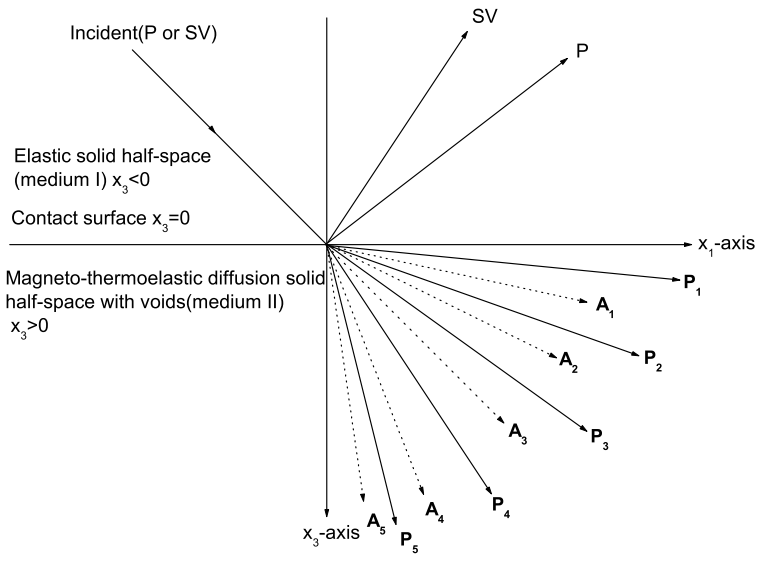

Fig. 1. Geometry of the problem

Using equations (1)-(3) and (19)-(21) in equation (15), we obtain

$$
\begin{aligned}
& F_{1}=\mu_{0} H_{0}^{2} \frac{\partial e^{*}}{\partial x}-\varepsilon_{0} \mu_{0}^{2} H_{0}^{2} \ddot{u}_{1}, \\
& F_{2}=0, \\
& F_{3}=\mu_{0} H_{0}^{2} \frac{\partial e^{*}}{\partial z}-\varepsilon_{0} \mu_{0}^{2} H_{0}^{2} \ddot{u}_{3} .
\end{aligned}
$$

We define the following dimensionless quantities

$$
\begin{array}{r}
x_{1}^{\prime}=\frac{w_{1}^{*} x_{1}}{c_{1}}, x_{3}^{\prime}=\frac{w_{1}^{*} x_{3}}{c_{1}}, \\
u_{1}^{\prime}=\frac{w_{1}^{*} u_{1}}{c_{1}}, u_{3}^{\prime}=\frac{w_{1}^{*} u_{3}}{c_{1}}, \\
u_{1}^{e^{\prime}}=\frac{w_{1}^{*} u_{1}^{e}}{c_{1}}, u_{3}^{e^{\prime}}=\frac{w_{1}^{*} u_{3}^{e}}{c_{1}},
\end{array}
$$

$$
\begin{array}{r}
t^{\prime}=w_{1}^{*} t, T^{\prime}=\frac{\beta_{1} T}{\rho c_{1}^{2}}, \\
C^{\prime}=\frac{\beta_{2} C}{\rho c_{1}^{2}}, \tau_{0}^{\prime}=w_{1}^{*} \tau_{0}, \\
\tau_{1}^{\prime}=w_{1}^{*} \tau_{1}, \\
\tau^{0^{\prime}}=w_{1}^{*} \tau^{0}, \tau^{1^{\prime}}=w_{1}^{*} \tau^{1},
\end{array}
$$




$$
\begin{array}{r}
\phi^{\prime}=\frac{\chi w_{1}^{* 2} \phi}{c_{1}^{2}}, \quad \sigma_{i j}^{\prime}=\frac{\sigma_{i j}}{\beta_{1} T_{0}}, \quad \sigma_{i j}^{e^{\prime}}=\frac{\sigma_{i j}^{e}}{\beta_{1} T_{0}}, \\
P_{i j}^{*^{\prime}}=\frac{P_{i j}^{*}}{\beta_{1} T_{0} c_{1}}, \quad P^{* e^{\prime}}=\frac{P^{* e}}{\beta_{1} T_{0} c_{1}},
\end{array}
$$

where $w_{1}^{*}=\frac{\rho C_{E} c_{1}^{2}}{K}, c_{1}=\sqrt{\frac{\lambda+2 \mu}{\rho}}$.

Upon introducing the quantities (23) in equations (11)(14) with the aid of (18) and (22) and after suppressing the primes, we obtain

$$
\begin{aligned}
& \delta_{1} \nabla^{2} u_{1}+\delta_{2} e_{, 1}^{*}+\delta_{3} \phi_{, 1}-\tau_{t}^{1} T_{, 1}-\tau_{c}^{1} C_{, 1}= \\
&=\left(1+\frac{\varepsilon_{0} \mu_{0}^{2} H_{0}^{2}}{\rho}\right) \ddot{u}_{1}, \\
& \delta_{1} \nabla^{2} u_{3}+\delta_{2} e_{, 3}^{*}+\delta_{3} \phi_{, 3}-\tau_{t}^{1} T_{, 3}-\tau_{c}^{1} C_{, 3}= \\
&=\left(1+\frac{\varepsilon_{0} \mu_{0}^{2} H_{0}^{2}}{\rho}\right) \ddot{u}_{3}, \\
&\left(\delta_{5} \nabla^{2}-\delta_{6}\right) \phi-\delta_{4} e^{*}+\delta_{7} \tau_{t}^{1} T+\delta_{8} \tau_{c}^{1} C=\ddot{\phi}, \\
& \zeta_{2} \tau_{e}^{0} \dot{e}^{*}+\zeta_{3} \tau_{e}^{0} \dot{\phi}+\tau_{t}^{0} \dot{T}+\zeta_{1} \tau_{c}^{0} \dot{C}=\nabla^{2} T, \\
& q_{1}^{*} \nabla^{2} e^{*}+q_{4}^{*} \nabla^{2} \phi+q_{2}^{*} \tau_{t}^{1} \nabla^{2} T-q_{3}^{*} \tau_{c}^{1} \nabla^{2} C+\tau_{f}^{0} \dot{C}=0,
\end{aligned}
$$

where

$$
\begin{gathered}
\delta_{1}=\frac{\mu}{\lambda+2 \mu}, \quad \delta_{2}=\frac{\lambda+\mu+\mu_{0} H_{0}^{2}}{\lambda+2 \mu}, \\
\delta_{3}=\frac{\gamma^{*}}{\rho \chi w_{1}^{* 2}}, \quad \delta_{4}=\frac{\gamma^{*}}{\lambda+2 \mu}, \quad \delta_{5}=\frac{a^{*}}{\chi(\lambda+2 \mu)}, \\
\delta_{6}=\frac{d^{*}}{\rho \chi w_{1}^{* 2}}, \delta_{7}=\frac{\xi^{*}}{\beta_{1}}, \delta_{8}=\frac{\zeta^{*}}{\beta_{2}}, \\
\zeta_{1}=\frac{a T_{0} c_{1}^{2} \beta_{1}}{w_{1}^{*} K \beta_{2}}, \zeta_{2}=\frac{\beta_{1}^{2} T_{0}}{\rho K w_{1}^{*}}, \zeta_{3}=\frac{\xi^{*} \beta_{1} T_{0} c_{1}^{2}}{\rho \chi K w_{1}^{* 3}} \\
q_{1}^{*}=\frac{D w_{1}^{*} \beta_{2}^{2}}{\rho c_{1}^{4}}, \quad q_{2}^{*}=\frac{D w_{1}^{*} \beta_{2} a}{\beta_{1} c_{1}^{2}} \\
q_{3}^{*}=\frac{D w_{1}^{*} b}{c_{1}^{2}}, \quad q_{4}^{*}=\frac{D \zeta^{*} \beta_{2}}{\rho \chi w_{1}^{*} c_{1}^{2}}, \\
\tau_{t}^{1}=1+\tau_{1} \frac{\partial}{\partial t}, \quad \tau_{c}^{1}=1+\tau^{1} \frac{\partial}{\partial t}, \\
\tau_{t}^{0}=1+\tau_{0} \frac{\partial}{\partial t}, \quad \tau_{c}^{0}=1+\gamma \frac{\partial}{\partial t}, \\
\tau_{e}^{0}=1+\varepsilon \tau_{0} \frac{\partial}{\partial t}, \quad \tau_{f}^{0}=1+\varepsilon \tau^{0} \frac{\partial}{\partial t}, \quad \nabla^{2} \equiv \frac{\partial^{2}}{\partial x_{1}^{2}}+\frac{\partial^{2}}{\partial x_{3}^{2}} .
\end{gathered}
$$

We introduce the potential functions $\hat{\phi}$ and $\hat{\psi}$ through the relations

$$
u_{1}=\frac{\partial \hat{\phi}}{\partial x_{1}}-\frac{\partial \hat{\psi}}{\partial x_{3}}, u_{3}=\frac{\partial \hat{\phi}}{\partial x_{3}}+\frac{\partial \hat{\psi}}{\partial x_{1}}
$$

where $\hat{\phi}$ and $\hat{\psi}$ are the displacement potentials of longitudinal and transverse waves.

Substituting equation (29) in the equations (24)-(28), we obtain

$$
\begin{gathered}
\delta_{9} \nabla^{2} \hat{\phi}-\delta_{10} \ddot{\hat{\phi}}=-\delta_{3} \phi+\tau_{t}^{1} T+\tau_{c}^{1} C, \\
\nabla^{2} \hat{\psi}=\frac{\delta_{10}}{\delta_{1}} \ddot{\hat{\psi}} \\
\left(\delta_{5} \nabla^{2}-\delta_{6}\right) \phi-\ddot{\phi}=\delta_{4} \nabla^{2} \hat{\phi}-\delta_{7} \tau_{t}^{1} T-\delta_{8} \tau_{c}^{1} C, \\
\nabla^{2} T=\tau_{t}^{0} \dot{T}+\zeta_{1} \tau_{c}^{0} \dot{C}+\zeta_{2} \tau_{e}^{0} \nabla^{2} \dot{\hat{\phi}}+\zeta_{3} \tau_{e}^{0} \dot{\phi}, \\
q_{3}^{*} \tau_{c}^{1} \nabla^{2} C=\tau_{f}^{0} \dot{C}+q_{1}^{*} \nabla^{4} \hat{\phi}+q_{2}^{*} \tau_{t}^{1} \nabla^{2} T+q_{4}^{*} \nabla^{2} \phi,
\end{gathered}
$$

where

$$
\delta_{9}=1+\frac{\mu_{0} H_{0}^{2}}{\lambda+2 \mu}, \delta_{10}=1+\frac{\varepsilon_{0} \mu_{0}^{2} H_{0}^{2}}{\rho} .
$$

Assuming the motion to be harmonic, we can write

$$
\{\hat{\phi}, \hat{\psi}, \phi, T, C\}\left(x_{1}, x_{3}, t\right)=\{\overline{\hat{\phi}}, \overline{\hat{\psi}}, \bar{\phi}, \bar{T}, \bar{C}\} e^{-\iota \omega t},
$$

where $\omega$ is the angular frequency of vibrations of material particles.

Substituting the expressions of $\hat{\phi}, \hat{\psi}, \phi, T, C$ given by equation (35) in the equations (30)-(34), we obtain

$$
\begin{gathered}
{\left[\delta_{9} \nabla^{2}+\delta_{10} \omega^{2}\right] \overline{\hat{\phi}}+\delta_{3} \bar{\phi}-\tau_{t}^{11} \bar{T}-\tau_{c}^{11} \bar{C}=0} \\
{\left[\nabla^{2}+\frac{\delta_{10} \omega^{2}}{\delta_{1}}\right] \overline{\hat{\psi}}=0} \\
\left(\delta_{5} \nabla^{2}-\delta_{6}+\omega^{2}\right) \bar{\phi}-\delta_{4} \nabla^{2} \overline{\hat{\phi}}+\delta_{7} \tau_{t}^{11} \bar{T}+\delta_{8} \tau_{c}^{11} \bar{C}=0 \\
-\zeta_{2} \tau_{e}^{10} \nabla^{2} \overline{\hat{\phi}}-\zeta_{3} \tau_{e}^{10} \bar{\phi}+\left[\nabla^{2}-\tau_{t}^{10}\right] \bar{T}-\zeta_{1} \tau_{c}^{10} \bar{C}=0 \\
q_{1}^{*} \nabla^{4} \overline{\hat{\phi}}+q_{4}^{*} \nabla^{2} \bar{\phi}+q_{2}^{*} \tau_{t}^{11} \nabla^{2} \bar{T}-\left[q_{3}^{*} \tau_{c}^{11} \nabla^{2}-\tau_{f}^{10}\right] \bar{C}=0
\end{gathered}
$$

where

$$
\begin{gathered}
\tau_{t}^{11}=1-\iota \omega \tau_{1}, \tau_{c}^{11}=1-\iota \omega \tau^{1}, \\
\tau_{t}^{10}=-\iota \omega\left(1-\iota \omega \tau_{0}\right), \tau_{c}^{10}=-\iota \omega(1-\iota \omega \gamma), \\
\tau_{e}^{10}=-\iota \omega\left(1-\iota \omega \varepsilon \tau_{0}\right), \tau_{f}^{10}=-\iota \omega\left(1-\iota \omega \varepsilon \tau^{0}\right) .
\end{gathered}
$$

Solving equations (36), (38)-(40), we obtain 


$$
\left[Z_{1} \nabla^{8}+Z_{2} \nabla^{6}+Z_{3} \nabla^{4}+Z_{4} \nabla^{2}+Z_{5}\right] \overline{\hat{\phi}}=0,
$$

where

$$
\begin{aligned}
& Z_{1}=\left(q_{1}^{*}-q_{3}^{*} \delta_{9}\right) \delta_{5} \tau_{c}^{11} \\
Z_{2}= & -\tau_{c}^{11}\left(q_{1}^{*}-q_{3}^{*} \delta_{9}\right)\left(\delta_{5} \tau_{t}^{10}+\delta_{6}-\omega^{2}\right) \\
+ & \zeta_{1} \delta_{5} \tau_{c}^{10} \tau_{t}^{11}\left(q_{1}^{*}+q_{2}^{*} \delta_{9}\right)+\zeta_{2} \delta_{5} \tau_{e}^{10} \tau_{t}^{11} \tau_{c}^{11}\left(q_{2}^{*}+q_{3}^{*}\right) \\
- & \delta_{5}\left(q_{3}^{*} \tau_{c}^{11} \delta_{10} \omega^{2}-\tau_{f}^{10} \delta_{9}\right)+\delta_{8} \tau_{c}^{11}\left(q_{1}^{*} \delta_{3}-q_{4}^{*}\right) \\
- & \delta_{4} \tau_{c}^{11}\left(q_{3}^{*} \delta_{3}-q_{4}^{*}\right), \\
Z_{3}= & \left(q_{3}^{*} \tau_{t}^{10} \tau_{c}^{11}+\tau_{f}^{10}+\zeta_{1} \tau_{c}^{10} q_{2}^{*} \tau_{t}^{11}\right)\left(\left(\delta_{10} \delta_{5}+\delta_{9}\right) \omega^{2}\right. \\
& \left.+\delta_{3} \delta_{4}-\delta_{6} \delta_{9}\right)-\tau_{t}^{11} \tau_{c}^{11} \tau_{e}^{10}\left(q_{2}^{*} \delta_{8}+q_{3}^{*} \delta_{7}\right) \\
& \times\left(-\zeta_{2} \delta_{3}+\zeta_{3} \delta_{9}\right) \\
& +\left(q_{1}^{*} \delta_{3}-q_{4}^{*} \delta_{9}\right)\left(\delta_{7} \zeta_{1} \tau_{c}^{10} \tau_{t}^{11}-\delta_{8} \tau_{c}^{11} \tau_{t}^{10}\right) \\
& +\delta_{10} \omega^{2} \tau_{c}^{11}\left(\left(\delta_{6}-\omega^{2}\right) q_{3}^{*}-\delta_{8} q_{4}^{*}\right) \\
& -\tau_{e}^{10} \tau_{t}^{11} \tau_{c}^{11}\left(-q_{4}^{*} \zeta_{2}+q_{1}^{*} \zeta_{3}\right)\left(\delta_{8}-\delta_{7}\right) \\
& +\left(q_{4}^{*} \delta_{4}-q_{1}^{*}\left(\delta_{6}-\omega^{2}\right)\right)\left(\zeta_{1} \tau_{c}^{10} \tau_{t}^{11}-\tau_{c}^{11} \tau_{t}^{10}\right) \\
& -\tau_{e}^{10} \tau_{t}^{11} \tau_{c}^{11}\left(-\zeta_{3} \delta_{4}+\zeta_{2}\left(\delta_{6}-\omega^{2}\right)\right)\left(q_{2}^{*}+q_{3}^{*}\right) \\
& -\tau_{f}^{10} \delta_{5}\left(\zeta_{2} \tau_{e}^{10} \tau_{t}^{11}+\tau_{t}^{10} \delta_{9}\right), \\
& \\
Z_{4}= & -\tau_{f}^{10} \tau_{t}^{10}\left(\left(\delta_{10} \delta_{5}+\delta_{9}\right) \omega^{2}+\delta_{3} \delta_{4}-\delta_{6} \delta_{9}\right) \\
+ & \tau_{e}^{10} \tau_{f}^{10} \tau_{t}^{11}\left[\zeta_{3}\left(\delta_{9} \delta_{7}-\delta_{4}\right)-\zeta_{2}\left(\delta_{7} \delta_{3}-\delta_{6}+\omega^{2}\right)\right] \\
- & \delta_{10} \omega^{2}\left[\left(\delta_{6}-\omega^{2}\right) \times\left(q_{3}^{*} \tau_{t}^{10} \tau_{c}^{11}+\tau_{f}^{10}+\zeta_{1} \tau_{c}^{10} \tau_{t}^{11} q_{2}^{*}\right)\right. \\
+ & \zeta_{3} \tau_{t}^{11} \tau_{c}^{11} \tau_{e}^{10}\left(\delta_{8} q_{2}^{*}+\delta_{7} q_{3}^{*}\right) \\
+ & \left.q_{4}^{*}\left(\delta_{7} \zeta_{1} \tau_{c}^{10} \tau_{t}^{11}-\delta_{8} \tau_{c}^{11} \tau_{t}^{10}\right)\right] \\
& Z_{5}=\tau_{f}^{10} \delta_{10} \omega^{2}\left[\left(\delta_{6}-\omega^{2}\right) \tau_{t}^{10}+\delta_{7} \zeta_{3} \tau_{e}^{10} \tau_{t}^{11}\right] . \\
& =
\end{aligned}
$$

The general solution of equation (41) can be written as

$$
\bar{\phi}=\bar{\phi}_{1}+\bar{\phi}_{2}+\bar{\phi}_{3}+\bar{\phi}_{4},
$$

where the potentials $\bar{\phi}_{i}, i=1,2,3,4$ are solutions of wave equations, given by

$$
\left[\nabla^{2}+\frac{\omega^{2}}{V_{i}^{2}}\right] \bar{\phi}_{i}=0, i=1,2,3,4 .
$$

Here $V_{1}, V_{2}, V_{3}$ and $V_{4}$ are the velocities of four longitudinal waves, that is, $\mathrm{P}, \mathrm{MD}$ (Mass Diffusive), T(Thermal) and $\mathrm{VF}$ (Volume fraction) waves and derived from the roots of biquadratic equation in $V^{2}$, given by

$$
Z_{5} V^{8}-Z_{4} \omega^{2} V^{6}+Z_{3} \omega^{4} V^{4}-Z_{2} \omega^{6} V^{2}+Z_{1} \omega^{8}=0 .
$$

From equation (37), we obtain

$$
\left[\nabla^{2}+\frac{\omega^{2}}{V_{5}^{2}}\right] \bar{\psi}=0,
$$

where $V_{5}=\sqrt{\frac{\delta_{1}}{\delta_{10}}}$ is the velocity of SV wave.

The general solutions for $\hat{\phi}, \phi, T$ and $C$ are obtained as

$$
\{\hat{\phi}, \phi, T, C\}=\sum_{i=1}^{4}\left\{1, \hat{q}_{i}, \hat{r}_{i}, \hat{s}_{i}\right\} \hat{\phi}_{i},
$$

where

$$
\begin{aligned}
& \hat{q}_{i}=-\frac{\left[-X_{5} \omega^{6}+V_{i}^{2} \omega^{4} X_{6}-\omega^{2} V_{i}^{4} X_{7}\right]}{-\omega^{6} X_{1}+V_{i}^{2} \omega^{4} X_{2}-V_{i}^{4} \omega^{2} X_{3}+V_{i}^{6} X_{4}}, \\
& \hat{r}_{i}=\frac{\left[-X_{8} \omega^{6}+V_{i}^{2} \omega^{4} X_{9}-\omega^{2} V_{i}^{4} X_{10}\right]}{-\omega^{6} X_{1}+V_{i}^{2} \omega^{4} X_{2}-V_{i}^{4} \omega^{2} X_{3}+V_{i}^{6} X_{4}}, \\
& \hat{s}_{i}=-\frac{\left[X_{11} \omega^{8}-V_{i}^{2} \omega^{6} X_{12}+\omega^{4} V_{i}^{4} X_{13}\right]}{V_{i}^{2}\left[-\omega^{6} X_{1}+V_{i}^{2} \omega^{4} X_{2}-V_{i}^{4} \omega^{2} X_{3}+V_{i}^{6} X_{4}\right]}, \\
& i=1, \ldots, 4 \text {, } \\
& X_{1}=-\delta_{5} q_{3}^{*} \tau_{c}^{11}, \\
& X_{2}=-q_{3}^{*} \tau_{c}^{11}\left(-\delta_{6}+\omega^{2}\right) \\
& +\delta_{5}\left(\tau_{f}^{10}+q_{3}^{*} \tau_{c}^{11} \tau_{t}^{10}+q_{2}^{*} \tau_{t}^{11} \zeta_{1} \tau_{c}^{10}\right)-\delta_{8} q_{4}^{*} \tau_{c}^{11}, \\
& X_{3}=-\delta_{5} \tau_{f}^{10} \tau_{t}^{10} \\
& +\left(-\delta_{6}+\omega^{2}\right)\left(\tau_{f}^{10}+q_{3}^{*} \tau_{c}^{11} \tau_{t}^{10}+q_{2}^{*} \tau_{t}^{11} \zeta_{1} \tau_{c}^{10}\right) \\
& -\tau_{t}^{11} \tau_{c}^{11} \tau_{e}^{10} \zeta_{3}\left(q_{2}^{*} \delta_{8}+q_{3}^{*} \delta_{7}\right) \\
& -q_{4}^{*}\left(\delta_{7} \zeta_{1} \tau_{c}^{10} \tau_{t}^{11}-\delta_{8} \tau_{c}^{11} \tau_{t}^{10}\right) \\
& X_{4}=\left(\delta_{6}-\omega^{2}\right) \tau_{f}^{10} \tau_{t}^{10}+\delta_{7} \zeta_{3} \tau_{e}^{10} \tau_{f}^{10} \tau_{t}^{11}, \\
& X_{5}=\tau_{c}^{11}\left(\delta_{4} q_{3}^{*}-\delta_{8} q_{1}^{*}\right), \\
& X_{6}=-\delta_{4}\left(\tau_{f}^{10}+q_{3}^{*} \tau_{c}^{11} \tau_{t}^{10}+q_{2}^{*} \tau_{t}^{11} \zeta_{1} \tau_{c}^{10}\right) \\
& -\tau_{c}^{11} \tau_{t}^{11} \tau_{e}^{10} \zeta_{2}\left(q_{2}^{*} \delta_{8}+q_{3}^{*} \delta_{7}\right) \\
& -q_{1}^{*}\left(\tau_{t}^{11} \zeta_{1} \tau_{c}^{10} \delta_{7}-\tau_{c}^{11} \tau_{t}^{10} \delta_{8}\right), \\
& X_{7}=\tau_{f}^{10}\left(\tau_{t}^{10} \delta_{4}+\zeta_{2} \tau_{e}^{10} \tau_{t}^{11} \delta_{7}\right), \\
& X_{8}=-\delta_{5} \zeta_{1}\left(q_{1}^{*} \tau_{c}^{10}+q_{3}^{*} \tau_{c}^{11} \tau_{e}^{10}\right), \\
& X_{9}=\tau_{c}^{11} \delta_{8} \tau_{e}^{10}\left(q_{1}^{*} \zeta_{3}-q_{4}^{*} \zeta_{2}\right) \\
& -\zeta_{1} \tau_{c}^{10}\left(q_{4}^{*} \delta_{4}+q_{1}^{*}\left(\omega^{2}-\delta_{6}\right)\right) \\
& -q_{3}^{*} \tau_{c}^{11} \tau_{e}^{10}\left(\zeta_{3} \delta_{4}+\zeta_{2}\left(\omega^{2}-\delta_{6}\right)\right)+\tau_{e}^{10} \tau_{f}^{10} \delta_{5} \zeta_{1}, \\
& X_{10}=\tau_{f}^{10} \tau_{e}^{10} \times\left(\zeta_{3} \delta_{4}+\zeta_{2}\left(\omega^{2}-\delta_{6}\right)\right),
\end{aligned}
$$




$$
\begin{gathered}
X_{11}=\delta_{5} q_{1}^{*} \tau_{t}^{10} \\
X_{12}=q_{4}^{*} \delta_{4}+q_{1}^{*}\left(\omega^{2}-\delta_{6}\right)+\delta_{5}\left(q_{2}^{*} \tau_{t}^{11} \zeta_{1} \tau_{e}^{10}-q_{1}^{*} \tau_{t}^{10}\right) \\
X_{13}=\delta_{7} \tau_{t}^{11} \tau_{e}^{10}\left(q_{1}^{*} \zeta_{3}-q_{4}^{*} \zeta_{2}\right) \\
+q_{2}^{*} \tau_{t}^{11} \tau_{e}^{10}\left(\zeta_{3} \delta_{4}+\zeta_{2}\left(\omega^{2}-\delta_{6}\right)\right)
\end{gathered}
$$

Applying the dimensionless quantities (23) in the equation (16) with the aid of (18) and after suppressing the primes, we obtain

$$
\begin{aligned}
& \frac{\beta^{e^{2}}}{c_{1}^{2}}\left[u_{1,11}^{e}+u_{1,33}^{e}\right]+\frac{\left(\alpha^{e^{2}}-\beta^{e^{2}}\right)}{c_{1}^{2}}\left[u_{1,11}^{e}+u_{3,13}^{e}\right]=\ddot{u}_{1}^{e}, \\
& \frac{\beta^{e^{2}}}{c_{1}^{2}}\left[u_{3,11}^{e}+u_{3,33}^{e}\right]+\frac{\left(\alpha^{e^{2}}-\beta^{e^{2}}\right)}{c_{1}^{2}}\left[u_{1,13}^{e}+u_{3,33}^{e}\right]=\ddot{u}_{3}^{e},
\end{aligned}
$$

where $\alpha^{e}=\sqrt{\frac{\lambda^{e}+2 \mu^{e}}{\rho^{e}}}, \beta^{e}=\sqrt{\frac{\mu^{e}}{\rho^{e}}}$ are velocities of longitudinal and transverse waves corresponding to medium I respectively.

The components $u_{1}^{e}$ and $u_{3}^{e}$ are related by the potential functions as

$$
u_{1}^{e}=\frac{\partial \hat{\phi}^{e}}{\partial x_{1}}-\frac{\partial \hat{\psi}^{e}}{\partial x_{3}}, u_{3}^{e}=\frac{\partial \hat{\phi}^{e}}{\partial x_{3}}+\frac{\partial \hat{\psi}^{e}}{\partial x_{1}},
$$

where $\hat{\phi}^{e}$ and $\hat{\psi}^{e}$ satisfy the wave equations as

$$
\nabla^{2} \hat{\phi}^{e}=\frac{\ddot{\hat{\phi}^{e}}}{\alpha^{\prime 2}}, \nabla^{2} \hat{\psi}^{e}=\frac{\ddot{\hat{\phi}}^{e}}{\beta^{\prime 2}},
$$

where $\alpha^{\prime}=\frac{\alpha^{e}}{c_{1}}$ and $\beta^{\prime}=\frac{\beta^{e}}{c_{1}}$.

\section{REFLECTION AND REFRACTION}

We consider a plane wave (P or SV) propagating through the isotropic elastic solid half-space and is incident at the interface $x_{3}=0$, as shown in Figure 1. Corresponding to this incident wave, two homogeneous waves (P and SV) are reflected in isotropic elastic solid half-space and five inhomogeneous waves (P, MD, T, VF and SV) are refracted in isotropic magneto-thermoelastic diffusion solid half-space with voids.

In elastic solid half-space, the potential functions satisfying equation (50) can be written as

$$
\begin{aligned}
\hat{\phi}^{e} & =A_{0}^{e} e^{\left[\imath \omega\left\{\left(x_{1} \sin \theta_{0}+x_{3} \cos \theta_{0}\right) / \alpha^{\prime}-t\right\}\right]} \\
& +A_{1}^{e} e^{\left[\iota \omega\left\{\left(x_{1} \sin \theta_{1}-x_{3} \cos \theta_{1}\right) / \alpha^{\prime}-t\right\}\right]}, \\
\hat{\psi}^{e} & =B_{0}^{e} e^{\left[\iota \omega\left\{\left(x_{1} \sin \theta_{0}+x_{3} \cos \theta_{0}\right) / \beta^{\prime}-t\right\}\right]} \\
& +B_{1}^{e} e^{\left[\iota \omega\left\{\left(x_{1} \sin \theta_{2}-x_{3} \cos \theta_{2}\right) / \beta^{\prime}-t\right\}\right]} .
\end{aligned}
$$

The coefficients $A_{0}^{e}\left(B_{0}^{e}\right), A_{1}^{e}$ and $B_{1}^{e}$ represent the amplitudes of the incident $\mathrm{P}($ or $\mathrm{SV})$, reflected $\mathrm{P}$ and reflected $\mathrm{SV}$ waves, respectively.

Following Borcherdt [42], in isotropic magneto-thermoelastic diffusive solid half-space with voids, the potential functions satisfying equations (43) and (45) can be written as

$$
\begin{gathered}
\{\hat{\phi}, \phi, T, C\}=\sum_{i=1}^{4}\left\{1, \hat{q}_{i}, \hat{r}_{i}, \hat{s}_{i}\right\} B_{i} e^{\left(\vec{A}_{i} \cdot \vec{r}\right)} e^{\left\{\iota\left(\vec{P}_{i} \cdot \vec{r}-\omega t\right)\right\}} \\
\hat{\psi}=B_{5} e^{\left(\vec{A}_{5} \cdot \vec{r}\right)} e^{\left\{\iota\left(\vec{P}_{5} \cdot \vec{r}-\omega t\right)\right\}}
\end{gathered}
$$

The coefficients $B_{i}, i=1, \ldots, 5$ represent the amplitudes of refracted P, MD, T, VF and SV waves, respectively. The propagation vector $\vec{P}_{i}, i=1, \ldots, 5$ and attenuation factor $\vec{A}_{i}, i=1, \ldots ., 5$ are given by

$$
\begin{array}{r}
\vec{P}_{i}=\xi_{R} \hat{x}_{1}+d V_{i R} \hat{x}_{3}, \\
\vec{A}_{i}=-\xi_{I} \hat{x}_{1}-d V_{i I} \hat{x}_{3}, \\
i=1, \ldots,, 5
\end{array}
$$

where

$$
\begin{gathered}
d V_{i}=d V_{i R}+\iota d V_{i I}=p \cdot v \cdot\left(\frac{\omega^{2}}{V_{i}^{2}}-\xi^{2}\right)^{1 / 2}, \\
i=1, \ldots, 5 .
\end{gathered}
$$

and $\xi=\xi_{R}+\iota \xi_{I}$ is a complex wave number. The subscripts $\mathrm{R}$ and I denote the real and imaginary parts of the corresponding complex quantity and p.v. stands for the principal value of the complex quantity obtained after square root. $\xi_{R} \geq 0$ ensures propagation in the positive $x_{1}$-direction. The complex wave number $\xi$ in the isotropic thermoelastic diffusion solid medium is given by

$$
\xi=\left|\vec{P}_{i}\right| \sin \theta_{i}^{\prime}-\iota\left|\vec{A}_{i}\right| \sin \left(\theta_{i}^{\prime}-\gamma_{i}\right), i=1, \ldots, 5,
$$

where $\gamma_{i}, i=1, \ldots, 5$ is the angle between the propagation and attenuation vector and $\theta_{i}^{\prime}, i=1, \ldots, 5$ is the angle of refraction in medium II.

\section{BOUNDARY CONDITIONS}

The boundary conditions to be satisfied at the interface $x_{3}=0$ are:

(i) Continuity of stress components

$$
\begin{gathered}
\sigma_{33}^{e}=\sigma_{33}, \\
\sigma_{31}^{e}=\sigma_{31},
\end{gathered}
$$

(ii) Continuity of displacement components

$$
u_{1}^{e}=u_{1}
$$




$$
u_{3}^{e}=u_{3},
$$

(iii) Thermally insulated boundary

$$
\frac{\partial T}{\partial x_{3}}=0
$$

(iv) Impermeable boundary

$$
\frac{\partial C}{\partial x_{3}}=0
$$

(v) Void Condition

$$
\frac{\partial \phi}{\partial x_{3}}=0
$$

Making use of potentials given by equations (51)-(54), we find that the boundary conditions are satisfied if and only if

$$
\xi_{R}=\frac{\omega \sin \theta_{0}}{V_{0}}=\frac{\omega \sin \theta_{1}}{\alpha^{\prime}}=\frac{\omega \sin \theta_{2}}{\beta^{\prime}},
$$

and

$$
\xi_{I}=0
$$

where

$$
V_{0}=\left\{\begin{array}{l}
\alpha^{\prime}, \text { for incident } \mathrm{P}-\text { wave } \\
\beta^{\prime}, \text { for incident } \mathrm{SV}-\text { wave }
\end{array}\right.
$$

It means that waves are attenuating only in $x_{3}$-direction. From equation (57), it implies that if $\left|\vec{A}_{i}\right| \neq 0$, then $\gamma_{i}=\theta_{i}^{\prime}, i=1, \ldots, 5$, that is, attenuated vectors for the four refracted waves are directed along the $x_{3}$-axis.

Using equations (51)-(54) in the boundary conditions (58)-(64), with the aid of equations (29), (49), (65)-(67), we obtain a system of seven non-homogeneous equations which can be written as

$$
\sum_{j=1}^{7} \hat{a}_{i j} R_{j}^{*}=S_{i}^{*}
$$

where $R_{j}^{*}=\left|R_{j}^{*}\right| e^{\iota \psi_{j}^{*}},\left|R_{j}^{*}\right|, \psi_{j}^{*}, j=1, \ldots, 7$ represent amplitudes ratios and phase shift of reflected P-, reflected SV-, refracted $\mathrm{P}-$, refracted $\mathrm{MD}-$, refracted T-, refracted $\mathrm{VF}$ - and refracted SV-waves to that of incident wave, respectively, and

$$
\begin{gathered}
\hat{a}_{11}=2 \mu^{e}\left(\frac{\xi_{R}}{\omega}\right)^{2}-\rho^{e} c_{1}^{2}, \quad \hat{a}_{12}=2 \mu^{e} \frac{\xi_{R}}{\omega} \frac{d V_{\beta^{\prime}}}{\omega}, \\
\hat{a}_{17}=2 \mu \frac{\xi_{R}}{\omega} \frac{d V_{5}}{\omega}, \quad \hat{a}_{21}=2 \mu^{e} \frac{\xi_{R}}{\omega} \frac{d V_{\alpha^{\prime}}}{\omega} \\
\hat{a}_{22}=\mu^{e}\left[\left(\frac{d V_{\beta^{\prime}}}{\omega}\right)^{2}-\left(\frac{\xi_{R}}{\omega}\right)^{2}\right], \quad \hat{a}_{27}=\mu\left[\left(\frac{\xi_{R}}{\omega}\right)^{2}-\left(\frac{d V_{5}}{\omega}\right)^{2}\right], \\
\hat{a}_{31}=\frac{\xi_{R}}{\omega}, \quad \hat{a}_{32}=\frac{d V_{\beta^{\prime}}}{\omega},
\end{gathered}
$$

$$
\begin{gathered}
\hat{a}_{37}=\frac{d V_{5}}{\omega}, \quad \hat{a}_{41}=-\frac{d V_{\alpha^{\prime}}}{\omega}, \quad \hat{a}_{42}=\frac{\xi_{R}}{\omega} \\
\hat{a}_{47}=-\frac{\xi_{R}}{\omega}, \quad \hat{a}_{51}=\hat{a}_{52}=\hat{a}_{57}=0 \\
\hat{a}_{61}=\hat{a}_{62}=\hat{a}_{67}=0, \hat{a}_{71}=\hat{a}_{72}=\hat{a}_{77}=0 \\
\hat{a}_{1 j}=\lambda\left(\frac{\xi_{R}}{\omega}\right)^{2}+\rho c_{1}^{2}\left(\frac{d V_{j}}{\omega}\right)^{2}+\frac{\rho c_{1}^{2}\left(\hat{q}_{j} \tau_{t}^{11}+\hat{r}_{j} \tau_{c}^{11}+\hat{s}_{j} \delta_{3}\right)}{\omega^{2}}, \\
\hat{a}_{2 j}=2 \mu \frac{\xi_{R}}{\omega} \frac{d V_{j}}{\omega}, \hat{a}_{3 j}=-\frac{\xi_{R}}{\omega}, \hat{a}_{4 j}=-\frac{d V_{j}}{\omega}, \\
\hat{a}_{5 j}=\hat{q}_{j} \frac{d V_{j}}{\omega}, \hat{a}_{6 j}=\hat{r}_{j} \frac{d V_{j}}{\omega}, \hat{a}_{7 j}=\hat{s}_{j} \frac{d V_{j}}{\omega}, j=3, \ldots, 6 \\
\frac{d V_{\alpha^{\prime}}}{\omega}=\left(\frac{1}{\alpha^{\prime 2}}-\left(\frac{\xi_{R}}{\omega}\right)^{2}\right)^{1 / 2}=\left(\frac{1}{\alpha^{\prime 2}}-\frac{\sin ^{2} \theta_{0}}{V_{0}^{2}}\right)^{1 / 2}, \\
\frac{d V_{\beta^{\prime}}}{\omega}=\left(\frac{1}{\beta^{\prime 2}}-\frac{\sin ^{2} \theta_{0}}{V_{0}^{2}}\right)^{1 / 2},
\end{gathered}
$$

and

$$
\frac{d V_{j}}{\omega}=p . v \cdot\left(\frac{1}{V_{j}^{2}}-\frac{\sin ^{2} \theta_{0}}{V_{0}^{2}}\right)^{1 / 2}, j=1, \ldots, 5 .
$$

Here p.v. is evaluated with restriction $d V_{j I} \geq 0$ to satisfy decay condition in magneto-thermoelastic diffusion medium with voids. The coefficients $S_{i}^{*}, i=1, \ldots, 7$ on right side of the equation (68) are given by

(i) For incident P-wave

$$
\begin{gathered}
S_{1}^{*}=-\hat{a}_{11}, \quad S_{2}^{*}=\hat{a}_{21}, \quad S_{3}^{*}=-\hat{a}_{31}, \\
S_{4}^{*}=\hat{a}_{41}, \quad S_{5}^{*}=0, \quad S_{6}^{*}=0, \quad S_{7}^{*}=0 .
\end{gathered}
$$

(ii) For incident SV-wave

$$
\begin{gathered}
S_{1}^{*}=\hat{a}_{12}, \quad S_{2}^{*}=-\hat{a}_{22}, \quad S_{3}^{*}=\hat{a}_{32}, \\
S_{4}^{*}=-\hat{a}_{42}, \quad S_{5}^{*}=0, \quad S_{6}^{*}=0, \quad S_{7}^{*}=0 .
\end{gathered}
$$

Now we consider a surface element of the unit area at the interface between two media. The purpose is to calculate the partition of energy of the incident wave among the reflected and refracted waves on the both sides of surface. Following Achenbach [43], energy flux across the surface element, that is, rate at which the energy is communicated per unit area of the surface is represented as

$$
P^{*}=\sigma_{l m} n_{m} \dot{u}_{l}
$$

where $\sigma_{l m}$ is the stress tensor, $n_{m}$ are the direction cosines of the unit normal $\hat{\boldsymbol{n}}$ outward to the surface element and $\dot{u}_{l}$ are the components of the particle velocity.

The time average of $P^{*}$ over a period, denoted by $\left\langle P^{*}\right\rangle$, represents the average energy transmission per unit surface area per unit time. Thus, on the surface with normal along 
$x_{3}$-direction, the average energy intensities of the waves in the elastic solid half-space are given by

$$
\left\langle P^{* e}\right\rangle=\operatorname{Re}\langle\sigma\rangle_{31}^{e} \operatorname{Re}\left(\dot{u}_{1}^{e}\right)+\operatorname{Re}\langle\sigma\rangle_{33}^{e} \operatorname{Re}\left(\dot{u}_{3}^{e}\right) .
$$

Following Achenbach [43], for any two complex functions $f$ and $g$, we have

$$
\langle\operatorname{Re}(f) \operatorname{Re}(g)\rangle=\frac{1}{2} \operatorname{Re}(f \bar{g}) .
$$

The expressions for energy ratios $E_{i}, i=1,2$ for the reflected P- and reflected SV are given by

$$
E_{i}=-\frac{\left\langle P_{i}^{* e}\right\rangle}{\left\langle P_{0}^{* e}\right\rangle}, i=1,2,
$$

where

$$
\begin{aligned}
& \left\langle P_{1}^{* e}\right\rangle=\frac{\omega^{4} \rho^{e} c_{1}^{2}}{\alpha^{\prime}}\left|R_{1}^{*}\right|^{2} \operatorname{Re}\left(\cos \theta_{1}\right), \\
& \left\langle P_{2}^{* e}\right\rangle=\frac{\omega^{4} \rho^{e} c_{1}^{2}}{\beta^{\prime}}\left|R_{2}^{*}\right|^{2} \operatorname{Re}\left(\cos \theta_{2}\right),
\end{aligned}
$$

and

(i) For incident P-wave

$$
\left\langle P_{0}^{* e}\right\rangle=-\frac{\omega^{4} \rho^{e} c_{1}^{2}}{\alpha^{\prime}} \cos \theta_{0},
$$

(ii) For incident SV-wave

$$
\left\langle P_{0}^{* e}\right\rangle=-\frac{\omega^{4} \rho^{e} c_{1}^{2}}{\beta^{\prime}} \cos \theta_{0},
$$

are the average energy intensities of the reflected $\mathrm{P}-$, reflected $\mathrm{SV}$-, incident $\mathrm{P}$ - and incident $\mathrm{SV}$-waves respectively. In equation (74), negative sign is taken because the direction of reflected waves is opposite to that of incident wave.

For magneto-thermoelastic diffusion solid half-space with voids, the average energy intensities of the waves on the surface with normal along $x_{3}$-direction, are given by

$$
\left\langle P_{i j}^{*}\right\rangle=\operatorname{Re}\langle\sigma\rangle_{31}^{(i)} \operatorname{Re}\left(\dot{u}_{1}^{(j)}\right)+\operatorname{Re}\langle\sigma\rangle_{33}^{(i)} \operatorname{Re}\left(\dot{u}_{3}^{(j)}\right) .
$$

The expressions for energy ratios $E_{i j}, i, j=1, \ldots ., 5$ for the refracted P-, refracted MD-, refracted T-, refracted VF- and refracted $\mathrm{SV}$-waves are given by

$$
E_{i j}=\frac{\left\langle P_{i j}^{*}\right\rangle}{\left\langle P_{0}^{* e}\right\rangle}, i, j=1, \ldots, 5
$$

where

$$
\left\langle P_{i j}^{*}\right\rangle=-\omega^{4} \operatorname{Re}\left[\left\{2 \mu \frac{d V_{i}}{\omega} \frac{\xi_{R}}{\omega} \frac{\bar{\xi}_{R}}{\omega}+\left\{\lambda\left(\frac{\xi_{R}}{\omega}\right)^{2}+\rho c_{1}^{2}\left(\frac{d V_{i}}{\omega}\right)^{2}\right.\right.\right.
$$

$$
\left.\left.\left.+\frac{\rho c_{1}^{2}\left(\hat{q}_{i} \tau_{t}^{11}+\hat{r}_{i} \tau_{c}^{11}+\hat{s}_{j} \delta_{3}\right)}{\omega^{2}}\right\} \frac{d \bar{V}_{j}}{\omega}\right\} R_{i+2}^{*} \bar{R}_{j+2}^{*}\right],
$$

$\left\langle P_{i 5}^{*}\right\rangle=-\omega^{4} \operatorname{Re}\left[\left\{-2 \mu \frac{d V_{i}}{\omega} \frac{\xi_{R}}{\omega} \frac{d \bar{V}_{5}}{\omega}+\left\{\lambda\left(\frac{\xi_{R}}{\omega}\right)^{2}+\rho c_{1}^{2}\left(\frac{d V_{i}}{\omega}\right)^{2}\right.\right.\right.$

$$
\left.\left.\left.+\frac{\rho c_{1}^{2}\left(\hat{q}_{i} \tau_{t}^{11}+\hat{r}_{i} \tau_{c}^{11}+\hat{s}_{j} \delta_{3}\right)}{\omega^{2}}\right\} \frac{\bar{\xi}_{R}}{\omega}\right\} R_{i+2}^{*} \bar{R}_{7}^{*}\right]
$$

$$
\begin{aligned}
\left\langle P_{5 j}^{*}\right\rangle & =-\omega^{4} \operatorname{Re}\left[\left\{\mu\left(\left(\frac{\xi_{R}}{\omega}\right)^{2}-\left(\frac{d V_{5}}{\omega}\right)^{2}\right) \frac{\bar{\xi}_{R}}{\omega}\right.\right. \\
& \left.\left.+2 \mu \frac{\xi_{R}}{\omega} \frac{d V_{5}}{\omega} \frac{d V_{j}}{\omega}\right\} R_{7}^{*} \bar{R}_{j+2}^{*}\right],
\end{aligned}
$$

$$
\begin{aligned}
\left\langle P_{55}^{*}\right\rangle & =-\omega^{4} \operatorname{Re}\left[\left\{-\mu\left(\left(\frac{\xi_{R}}{\omega}\right)^{2}-\left(\frac{d V_{5}}{\omega}\right)^{2}\right) \frac{d \bar{V}_{5}}{\omega}\right.\right. \\
& \left.\left.+2 \mu \frac{\xi_{R}}{\omega} \frac{d V_{5}}{\omega} \frac{\bar{\xi}_{R}}{\omega}\right\} R_{7}^{*} \bar{R}_{7}^{*}\right], i, j=1, \ldots, 4 .
\end{aligned}
$$

The diagonal entries of energy matrix $E_{i j}$ in equation (78) represent the energy ratios of $\mathrm{P}, \mathrm{MD}, \mathrm{T}, \mathrm{VF}$ and $\mathrm{SV}$ waves, respectively, whereas the sum of the non-diagonal entries of $E_{i j}$ give the share of interaction energy among all the refracted waves in the medium and is given by

$$
E_{R R}=\sum_{i=1}^{5}\left(\sum_{j=1}^{5} E_{i j}-E_{i i}\right) .
$$

The energy ratios $E_{i}, i=1,2$, diagonal entries and sum of non-diagonal entries of energy matrix $E_{i j}$, that is, $E_{11}, E_{22}, E_{33}, E_{44}, E_{55}$ and $E_{R R}$ yield the conservation of incident energy across the interface, through the relation

$$
E_{1}+E_{2}+E_{11}+E_{22}+E_{33}+E_{44}+E_{55}+E_{R R}=1 .
$$

\section{PARTICULAR CASES}

1. In the absence of magnetic field, that is, we take $H_{0}=0$ in the equations (68) and (78), we obtain the results for amplitude and energy ratios of reflected $\mathrm{P}-$, reflected SV-, refracted P-, refracted MD-, refracted T-, refracted VF-and refracted SV-waves in thermoelastic diffusion solid half-space with voids to that of the incident wave.

2. In the absence of void effect, If we take $\gamma^{*}=\xi^{*}=$ $\zeta^{*}=0$, in the basic equations (11)-(14) and follow the similar procedure, we obtain the corresponding expressions for amplitude and energy ratios of reflected P-, reflected SV-, refracted $\mathrm{P}-$, refracted $\mathrm{MD}-$, refracted $\mathrm{T}$ - and refracted SVwaves to that of incident wave. In these expressions the velocities $V_{i}, i=1,2,3$ are derived from the roots of cubic equation in $V^{2}$, given by 


$$
Y_{4} V^{6}-Y_{3} \omega^{2} V^{4}+Y_{2} \omega^{4} V^{2}-Y_{1} \omega^{6}=0,
$$

where

$$
\begin{aligned}
Y_{1} & =\left(q_{1}^{*}-q_{3}^{*} \delta_{9}\right) \tau_{c}^{11} \\
Y_{2} & =\delta_{9} \tau_{f}^{10}+\left(q_{1}^{*}+q_{2}^{*} \delta_{9}\right) \zeta_{1} \tau_{c}^{10} \tau_{t}^{11} \\
& +\left(q_{2}^{*}+q_{3}^{*}\right) \zeta_{2} \tau_{t}^{11} \tau_{e}^{10} \tau_{c}^{11} \\
& -\left(q_{1}^{*}-q_{3}^{*} \delta_{9}\right) \tau_{t}^{10} \tau_{c}^{11}-q_{3}^{*} \delta_{10} \tau_{c}^{11} \omega^{2}, \\
Y_{3} & =\tau_{f}^{10}\left(\delta_{10} \omega^{2}-\delta_{9} \tau_{t}^{10}-\zeta_{2} \tau_{t}^{11} \tau_{e}^{10}\right)+q_{3}^{*} \delta_{10} \tau_{t}^{10} \tau_{c}^{11} \omega^{2} \\
& +q_{2}^{*} \delta_{10} \zeta_{1} \tau_{c}^{10} \tau_{t}^{11} \omega^{2}, \\
Y_{4} & =-\delta_{10} \omega^{2} \tau_{f}^{10} \tau_{t}^{10}
\end{aligned}
$$

and the coupling coefficients $\hat{q}_{i}, \hat{r}_{i} i=1,2,3$ are given as

$$
\begin{gathered}
\hat{q}_{i}=\frac{\left[q_{3}^{*} \tau_{c}^{11} \zeta_{2} \tau_{e}^{10}+q_{1}^{*} \zeta_{1} \tau_{c}^{10}\right] \omega^{4}+\zeta_{2} \tau_{e}^{10} \tau_{f}^{10} \omega^{2} V_{i}^{2}}{\tau_{t}^{10} \tau_{f}^{10} V_{i}^{4}+\left[q_{3}^{*} \tau_{c}^{11} \tau_{t}^{10}+\tau_{f}^{10}+q_{2}^{*} \tau_{t}^{11} \zeta_{1} \tau_{c}^{10}\right]}, \\
\times \omega^{2} V_{i}^{2}+q_{3}^{*} \tau_{c}^{11} \omega^{4} \\
\hat{r}_{i}=\frac{-q_{1}^{*} \omega^{6}+\left[q_{2}^{*} \tau_{t}^{11} \zeta_{2} \tau_{e}^{10}-q_{1}^{*} \tau_{t}^{10}\right] \omega^{4} V_{i}^{2}}{V_{i}^{2}\left[\tau_{t}^{10} \tau_{f}^{10} V_{i}^{4}+\left[q_{3}^{*} \tau_{c}^{11} \tau_{t}^{10}+\tau_{f}^{10}+q_{2}^{*} \tau_{t}^{11} \zeta_{1} \tau_{c}^{10}\right]\right.}, \\
\left.\times \omega^{2} V_{i}^{2}+q_{3}^{*} \tau_{c}^{11} \omega^{4}\right] \\
i=1,2,3 .
\end{gathered}
$$

3. In the absence of void and magnetic field, that is by taking $\gamma^{*}=\xi^{*}=\zeta^{*}=H_{0}=0$ in equations (68) and (78), the same results are obtained as in Kumar and Kansal [44].

\section{NUMERICAL RESULTS AND DISCUSSION}

With the view of illustrating theoretical results obtained in the preceding sections and comparing these in the context of various theories of thermoelastic diffusion, we now represent some numerical results for copper material [20], the physical data for which is given below:

$$
\begin{gathered}
\lambda=7.76 \times 10^{10} \mathrm{Kg} \mathrm{m}^{-1} \mathrm{~s}^{-2}, \\
\mu=3.86 \times 10^{10} \mathrm{Kg} \mathrm{m}^{-1} \mathrm{~s}^{-2}, \quad T_{0}=0.293 \times 10^{3} \mathrm{~K}, \\
C_{E}=.3831 \times 10^{3} \mathrm{JKg}^{-1} \mathrm{~K}^{-1}, \\
\alpha_{t}=1.78 \times 10^{-5} \mathrm{~K}^{-1}, \quad \alpha_{c}=1.98 \times 10^{-4} \mathrm{Kg}^{-1} \mathrm{~m}^{3}, \\
a=1.2 \times 10^{4} \mathrm{~m}^{2} \mathrm{~s}^{-2} \mathrm{~K}^{-1}, \quad b=9 \times 10^{5} \mathrm{Kg}^{-1} \mathrm{~m}^{5} \mathrm{~s}^{-2}, \\
D=0.85 \times 10^{-8} \mathrm{Kg} \mathrm{s} \mathrm{m}^{-3}, \quad \rho=8.954 \times 10^{3} \mathrm{Kg} \mathrm{m}^{-3}, \\
K=0.383 \times 10^{3} \mathrm{Wm}^{-1} \mathrm{~K}^{-1} .
\end{gathered}
$$

The relaxation times are:

$$
\tau_{0}=0.8 \mathrm{~s}, \tau_{1}=0.85 \mathrm{~s}, \tau^{0}=0.9 \mathrm{~s}, \tau^{1}=0.95 \mathrm{~s} .
$$

The values of $\delta_{9}$ and $\delta_{10}$ are taken as 1.8 and 1.7, respectively.

Following Bullen [45], the numerical data of granite in elastic medium is given by

$$
\begin{gathered}
\rho^{e}=2.65 \times 10^{3} \mathrm{Kg} \mathrm{m}^{-3}, \\
\alpha^{e}=5.27 \times 10^{3} \mathrm{~m} \mathrm{~s}^{-1}, \quad \beta^{e}=3.17 \times 10^{3} \mathrm{~m} \mathrm{~s}^{-1},
\end{gathered}
$$

The void parameters are

$$
\begin{gathered}
\chi=1.75 \times 10^{-15} \mathrm{~m}^{2}, \gamma^{*}=1.139 \times 10^{10} \mathrm{Kg} \mathrm{m}^{-1} \mathrm{~s}^{-2}, \\
a^{*}=3.688 \times 10^{-5} \mathrm{Kg} \mathrm{m} \mathrm{s}^{-2}, K g m s^{-2}, \\
d^{*}=1.475 \times 10^{10} \mathrm{Kg} \mathrm{m}^{-1} \mathrm{~s}^{-2}, \\
\xi^{*}=2.0 \times 10^{6} \mathrm{Kg} \mathrm{m}^{-1} \mathrm{~s}^{-2} \mathrm{~K}^{-1}, \zeta^{*}=2.9 \times 10^{6} \mathrm{~m}^{2} \mathrm{~s}^{-2} .
\end{gathered}
$$

The software Matlab 7.0.4 has been used to determine the values of energy ratios $E_{i}, i=1,2$ and an energy matrix $E_{i j}, i, j=1, \ldots ., 5$ defined in the previous section for different values of incident angle $\left(\theta_{0}\right)$ ranging from $0^{\circ}$ to $90^{\circ}$ for fixed frequency $\omega=2 \times \pi \times 2000 \mathrm{~Hz}$. For numerical purpose, only L-S theory of thermoelastic diffusion with voids has been considered. Corresponding to incident P and SV waves, the variations of these energy ratios with respect to angle of incidence have been plotted in Figs. 2-5 and Figs. 6-9, respectively. Due to negligible values of $E_{11}, E_{22}, E_{33}, E_{44}$ the values of these energy ratios are not shown in figures. In all the Figures, the vertical and horizontal lines correspond to theories of magneto-thermoelastic diffusion with voids (MTDV) and thermoelastic diffusion with voids (TDV), respectively.

\section{Incident $P$ wave}

It is clear from Figure 2 that for MTDV and TDV theories, the values of energy ratio $E_{1}$ decrease with the increase of the angle of incidence $\left(\theta_{0}\right)$ from $0^{\circ}$ to $69^{\circ}$ and $0^{\circ}$ to $75^{\circ}$, respectively, and then increase as $\theta_{0}$ increases further. Figure 3 shows that the values of energy ratio $E_{2}$ increase upto at $\theta_{0}=72^{\circ}$ and thereafter decrease continuously. From Figure 4, it is noticed that values of energy ratio $E_{55}$ increase to their highest values at $\theta=72^{\circ}$ and then decrease continuously. Figure 5 shows that initially the values of energy ratio $E_{R R}$ decrease, but finally increase slowly and steadily. It is noticed that the sum of the values of energy ratios $E_{1}, E_{2}, E_{11}, E_{22}, E_{33}, E_{44}, E_{55}$ and $E_{R R}$ is found to be exactly unity at each value of $\theta_{0}$ which proves the law of conservation of energy at the interface. However, if we see Figures 2-5 closely, we find that the sum does not looks to be unity. The reason is that we are plotting $3 \mathrm{D}$ graphs in origin software. On the other hand, if we plot 2D graphs in any other software, the sum will come out exactly unity.

\section{Incident $\mathrm{SV}$ wave}

From Figure 6, it is evident that there is a rapid increase in the values of energy ratio $E_{1}$ initially, but after $\theta=36^{\circ}$ 


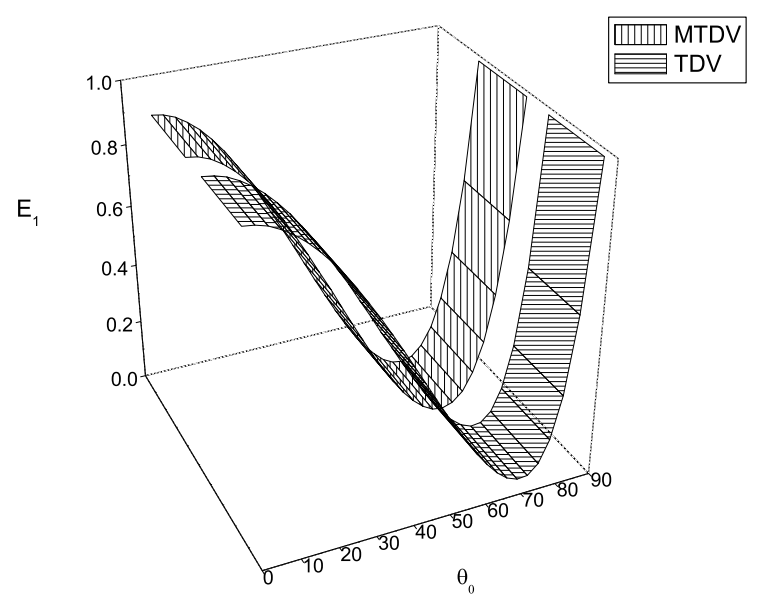

Fig. 2. Variations of energy ratio $\left(E_{1}\right)$ with respect to angle of incidence $\left(\theta_{0}\right)$ for P-wave

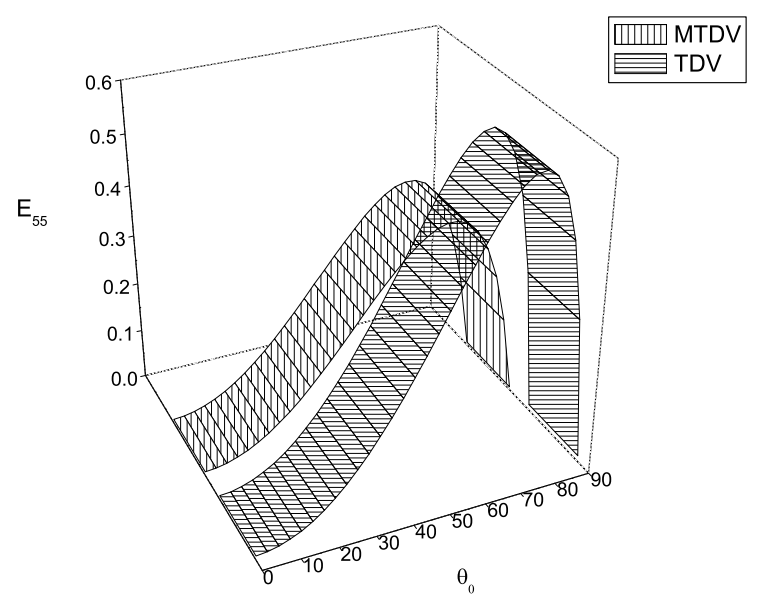

Fig. 4. Variations of energy ratio $\left(E_{55}\right)$ with respect to angle of incidence $\left(\theta_{0}\right)$ for P-wave

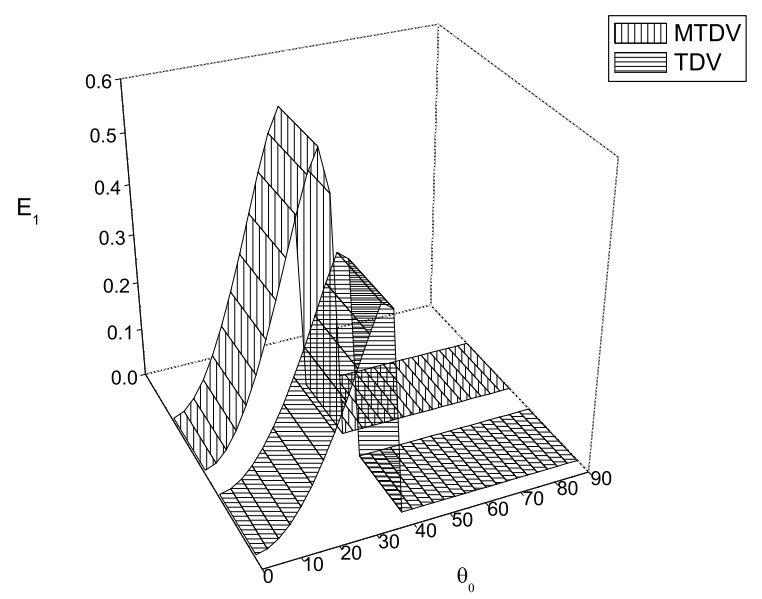

Fig. 6. Variations of energy ratio $\left(E_{1}\right)$ with respect to angle of incidence $\left(\theta_{0}\right)$ for $\mathrm{SV}$-wave

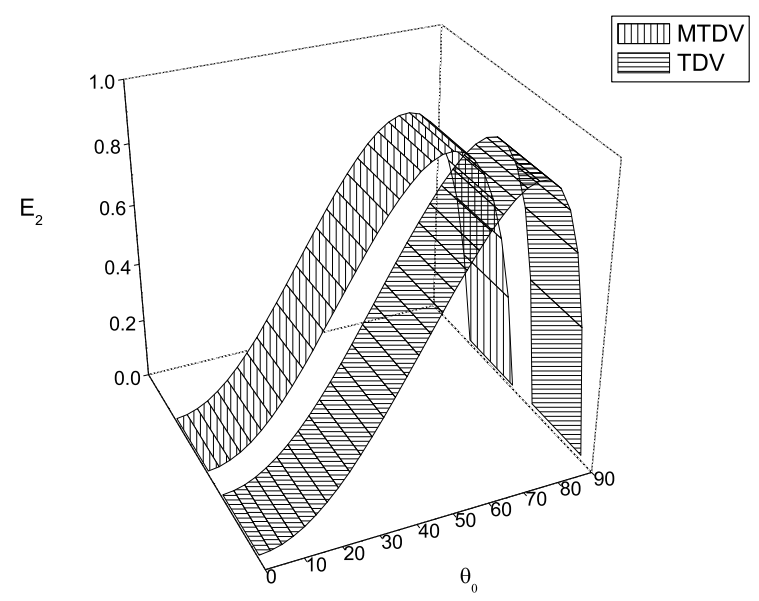

Fig. 3. Variations of energy ratio $\left(E_{2}\right)$ with respect to angle of incidence $\left(\theta_{0}\right)$ for $\mathrm{P}$-wave

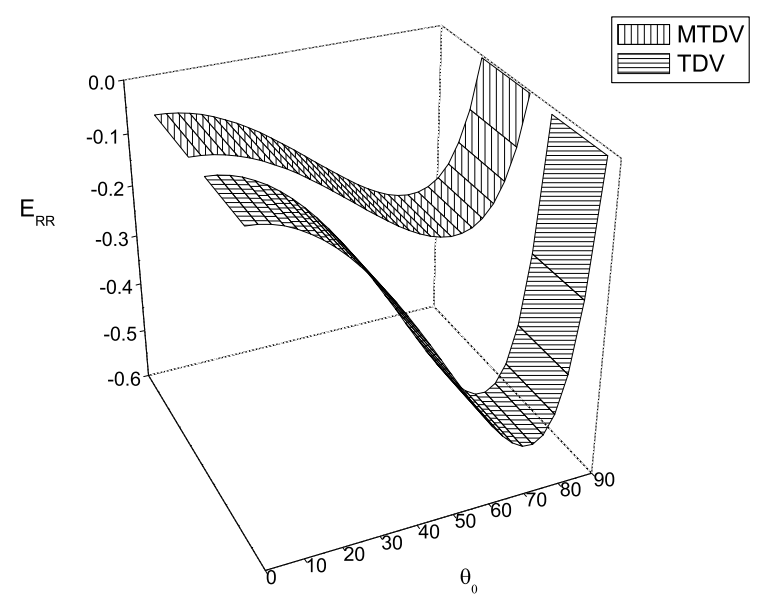

Fig. 5. Variations of energy ratio $\left(E_{R R}\right)$ with respect to angle of incidence $\left(\theta_{0}\right)$ for P-wave

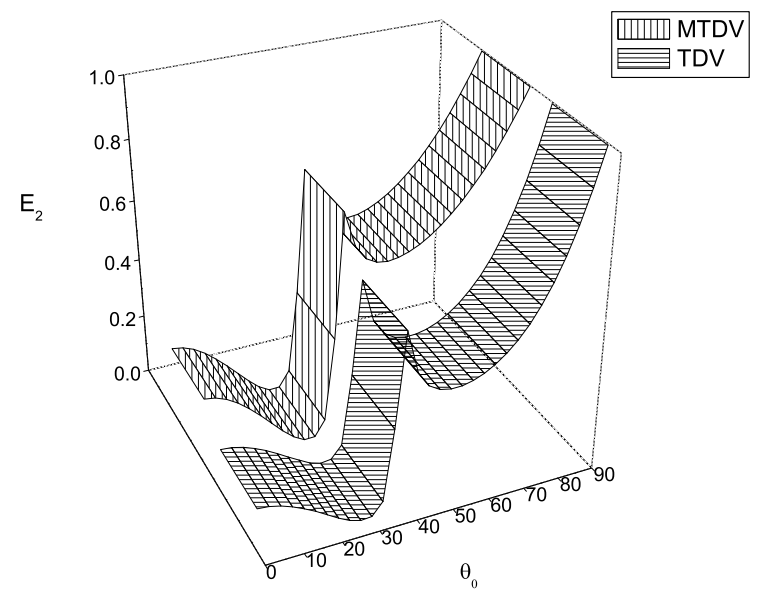

Fig. 7. Variations of energy ratio $\left(E_{2}\right)$ with respect to angle of incidence $\left(\theta_{0}\right)$ for $\mathrm{SV}$-wave 


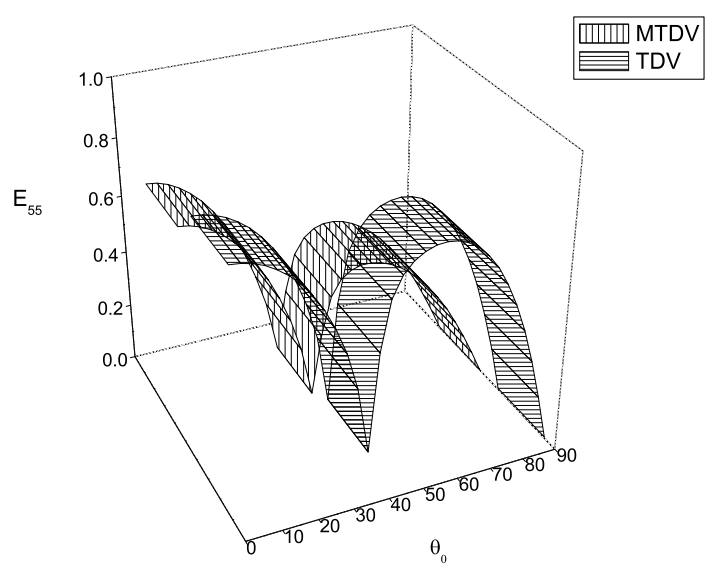

Fig. 8. Variations of energy ratio $\left(E_{55}\right)$ with respect to angle of incidence $\left(\theta_{0}\right)$ for $\mathrm{SV}$-wave

and onwards, values of energy ratio $E_{1}$ decrease and become negligible small. Figure 7 depicts that the values of energy ratio $E_{2}$ initially fluctuate, but finally reach to nearly unity. We notice from Figures 8 and 9 that firstly, the values of energy ratios $E_{55}$ and $E_{R R}$ show fluctuating behavior and then decrease and increase respectively. Like in case of incident $P$ wave, the sum of all energy ratios is also found to be unity in case of incident $\mathrm{SV}$ wave.

\section{CONCLUSIONS}

In the present article, the phenomenon of reflection and refraction of obliquely incident elastic waves at the interface between an elastic solid half-space and a magneto-thermoelastic diffusive solid half-space with voids has been studied. The five waves in magneto-thermoelastic diffusive medium with voids are identified and explained through different wave equations in terms of displacement potentials. Due to the presence of dissipation, the waves in magneto-thermoelastic diffusive medium with voids are considered to be inhomogeneous waves. The energy ratios of different reflected and refracted waves to that of the incident wave are computed numerically and presented graphically with respect to the angle of incidence.

From numerical results, we conclude that the effect of angle of incidence on the energy ratios of the reflected and refracted waves is significant. The sum of all energy ratios of the reflected waves, refracted waves and interference between refracted waves is verified to be always unity, which ensures the law of conservation of incident energy at the interface.

\section{References}

[1] H.W. Lord, Y. Shulman, A generalized dynamical theory of thermoelasticity, Journal of Mechanics and Physics of Solids 15, 299-309 (1967).

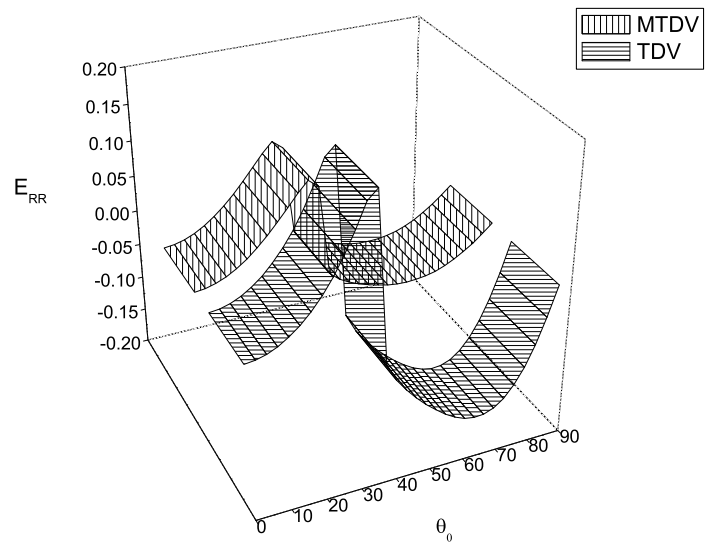

Fig. 9. Variations of energy ratio $\left(E_{R R}\right)$ with respect to angle of incidence $\left(\theta_{0}\right)$ for $\mathrm{SV}$-wave

[2] A.E. Green, K.A. Lindsay, Thermoelasticity, Journal of Elasticity 2, 1-7 (1972).

[3] R.S. Dhaliwal, H.H. Sherief, Generalized thermoelasticity for anisotropic media, Quarterly of Applied Mathematics 38, 1-8 (1980).

[4] D.S. Chanderashekhariah, Thermoelasticity with second sound: a review, Applied Mechanics Review 39, 355-376 (1986).

[5] R.B. Hetnarski, J. Ignaczak, Generalized thermoelasticity, Journal of Thermal Stresses 22, 451-476 (1999).

[6] J.W. Nunziato, S.C. Cowin, A non-linear theory of elastic material with voids, Archive for Rational Mechanics and Analysis 72, 175-201 (1979).

[7] S.C. Cowin, J.W. Nunziato, Linear theory of elastic materials with voids, Journal of Elasticity 13, 125-147 (1983).

[8] D. Iesan, A theory of thermoelastic materials with voids, Acta Mechanica 60, 67-89 (1986).

[9] M. Ciarletta, A. Scalia, On the nonlinear theory of nonsimple thermoelastic materials with voids, ZAMM 73, 7-75 (1993).

[10] W. Nowacki, Dynamical problems of thermodiffusion in solids-I, Bulletin of Polish Academy of Sciences Series, Science and Technology 22, 55-64 (1974a).

[11] W. Nowacki, Dynamical problems of thermodiffusion in solids-II, Bulletin of Polish Academy of Sciences Series, Science and Technology 22, 205-211 (1974b).

[12] W. Nowacki, Dynamical problems of thermodiffusion in solids-III, Bulletin of Polish Academy of Sciences Series, Science and Technology 22, 257-266 (1974c).

[13] W. Nowacki, Dynamical problems of diffusion in solids, Engineering Fracture Mechanics 8, 261-266 (1976).

[14] W. Dudziak, S.J. Kowalski, Theory of thermodiffusion for solids, International Journal of Heat and Mass Transfer 32, 2005-2013 (1989).

[15] Z.S. Olesiak, Y.A. Pyryev, A coupled quasi-stationary problem of thermodiffusion for an elastic cylinder, International Journal of Engineering Science 33, 773-780 (1995).

[16] J.A. Gawinecki, P. Kacprzyk, P. Bar-Yoseph, Initial boundary value problem for some coupled nonlinear parabolic system of partial differential equations appearing in thermoelastic diffusion in solid body, Journal for Analysis and its Applications 19, 121-130 (2000). 
[17] J.A. Gawinecki, A. Szymaniec, Global solution of the Cauchy problem in nonlinear thermoelastic diffusion in solid body, Proceedings in Applied Mathematics and Mechanics 1, 446447 (2002).

[18] H.H. Sherief, F.A. Hamza and H.A. Saleh, The theory of generalized thermoelastic diffusion, International Journal of Engineering Science 42, 591-608 (2004).

[19] M. Aouadi, Uniqueness and reciprocity theorems in the theory of generalized thermoelastic diffusion, Journal of Thermal Stresses 30, 665-678 (2007).

[20] H.H. Sherief, H.A. Saleh, A half space problem in the theory of generalized thermoelastic diffusion, International Journal of Solids and Structures 42, 4484-4493 (2005).

[21] R. Kumar, T. Kansal, Variational principle, uniqueness and reciprocity theorems in the theory of generalized thermoelastic diffusion material, QScience Connect 2013, 1-18 (2013).

[22] P. Borejko, Reflection and transmission coefficients for threedimensional plane waves in elastic media, Wave Motion 24, 371-393 (1996).

[23] C.M. Wu, B. Lundberg, Reflection and transmission of the energy of harmonic elastic waves in a bent bar, Journal of Sound and Vibration 190, 645-659 (1996).

[24] S.B. Sinha, K.A. Elsibai, Reflection and refraction of thermoelastic waves at an interface of two semi-infinite media with two relaxation times, Journal of Thermal Stresses $\mathbf{2 0}$, 129-145 (1997).

[25] M.D. Sharma, M.L. Gogna, Reflection and refraction of plane harmonic waves at an interface between elastic solid and porous solid saturated by viscous liquid, PAGEOPH 138, 249-266 (1992).

[26] S.K. Tomar, A. Arora, Reflection and transmission of elastic waves at an elastic/porous solid saturated by immiscible fluids, International Journal of Solids and Structures 43, 1991-2013 (2006).

[27] R. Kumar, P. Sarthi, Reflection and refraction of thermoelastic plane waves at an interface of two thermoelastic media without energy dissipation, Archives of Mechanics 58, 155185 (2006).

[28] A.N. Abd-alla, A.A.S. Al-dawy, The reflection phenomena of $S V$-waves in a generalized thermoelastic medium, International Journal of Mathematics and Mathematical Sciences 23, 529-546 (2000).

[29] M. Ciarletta, M.A. Sumbatyan, Reflection of plane waves by the free boundary of a porous elastic half-space, Journal of Sound and Vibration 259, 253-264 (2003).

[30] J. Singh, S.K. Tomar, Plane waves in thermo-elastic material with voids, Mechanics of Materials 39, 932-940 (2007).

[31] B. Singh, Wave propagation in a generalized thermoelastic material with voids, Applied Mathematics and Computation 189, 698-709 (2007).
[32] J. Singh, S.K. Tomar, Reflection and transmission of transverse waves at a plane interface between two different porous elastic half-spaces, Applied Mathematics and Computation 176, 364-378 (2006).

[33] N.C. Das, A. Lahiri, S. Sarkar and B. Basu, Reflection of generalized thermoelastic waves from isothermal and insulated boundaries of a half-space, Computers and Mathematics with Applications 56, 2795-2805 (2008).

[34] S.S. Singh, Reflection and transmission of couple longitudinal waves at a plane interface between two dissimilar halfspaces of thermo-elastic materials with voids, Applied Mathematics and Computation 218, 3359-3371 (2011).

[35] R. Kumar, R. Kumar, Reflection and transmission at the plane boundary of elastic half-space and initially stressed thermoelastic diffusion with voids half-space, MMMS 9, 185-213 (2013).

[36] A.N. Abd-alla, Relaxation effects on reflection of generalized magneto-thermoelastic waves, Mechanics Research Communications 27, 591-600 (2000).

[37] M.I.A. Othman, Y. Song, Reflection of magneto-thermoelastic waves with two relaxation times and temperature dependent elastic moduli, Applied Mathematics and Modelling 32, 483-500 (2008).

[38] M.I.A. Othman, R. Kumar, Reflection of magneto-thermoelasticity waves with temperature dependent properties in generalized thermoelasticity, International Communications in Heat and Mass Transfer 36, 513-520 (2009).

[39] S.M. Abo-Dahab, Propagation of $P$ waves from stress-free surface elastic half-space with voids under thermal relaxation and magnetic field, Applied Mathematics and Modelling 34, 1798-1806 (2010).

[40] M.I.A. Othman, Generalized electro-magneto-thermoelasticity in case of thermal shock plane waves for a finite conducting half-Space with two relaxation times, Mechanics and Mechanical Engineering 14, 5-30 (2010).

[41] B. Singh, L. Singh and S. Deswal, Reflection of plane waves from a free surface of a generalized magneto-thermoelastic solid half-space with diffusion, Journal of Theoretical and Applied Mechanics 52, 385-394 (2014).

[42] R.D. Borcherdt, Reflection-refraction of general P and type-I $S$ waves in elastic and anelastic solids, Geophysical Journal of Royal Astronomical Society 70, 621-638 (1982).

[43] J.D. Achenbach, Wave propagation in elastic solids, NorthHolland, Amsterdam 1973.

[44] R. Kumar, T. Kansal, Reflection and refraction of plane waves at an interface of an elastic solid half-space and a thermoelastic diffusive solidhalf-space, Archives of Mechanics 64, 293-317 (2012).

[45] K.E. Bullen, An introduction to the theory of Seismology, Cambridge University Press, 1963. 


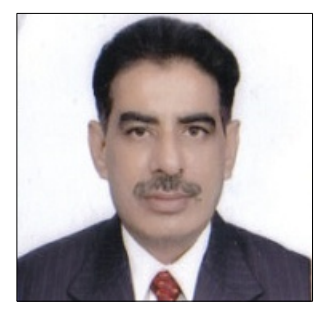

Rajneesh Kumar

Prof. Rajneesh Kumar is a retired Professor of Mathematics at Kurukshetra University, Kurukshetra, Haryana (India). His field of expertise is solid mechanics and thermoelasticity.

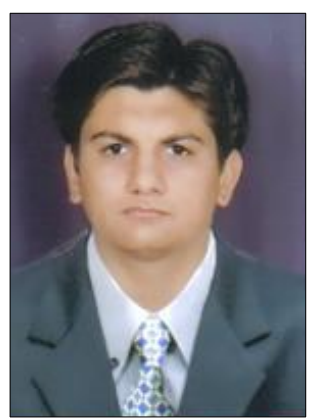

\section{Tarun Kansal}

Dr. Tarun Kansal is an Assistant Professor of Mathematics at M.N. College, Shahabad (M.), Kurukshetra, Haryana (India). His field of research is thermoelasticity. 